NASA Contractor Report 187517

ICASE Report No. 91-15

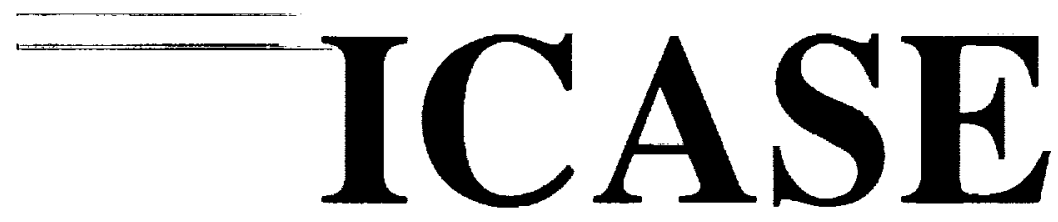

\title{
A FULLY GALERKIN METHOD FOR THE RECOVERY OF STIFFNESS AND DAMPING PARAMETERS IN EULER-BERNOULLI BEAM MODELS
}
R. C. Smith
K. L. Bowers

Contract No. NAS1-18605

February 1991

Institute for Computer Applications in Science and Engineering NASA Langley Research Center

Hampton, Virginia 23665-5225

Operated by the Universities Space Research Association

\section{N/SA \\ Nalional Aeronaulics and \\ Space Adminisiration}

Langley Research Center

Hamplon, Virginia 23665-52.25

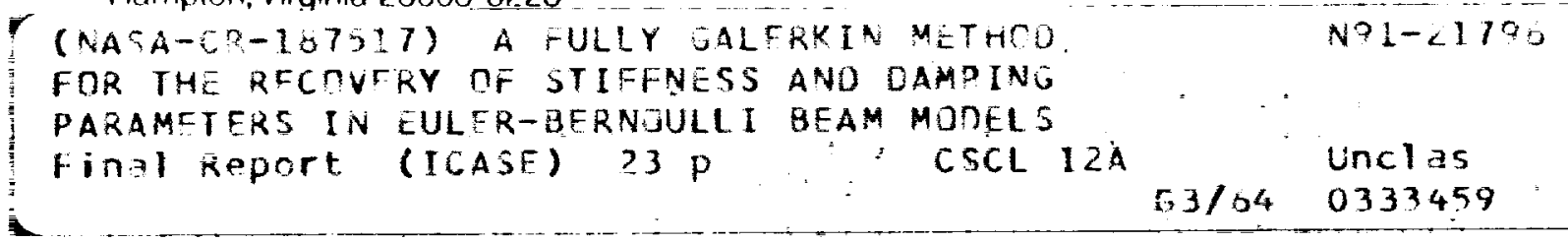




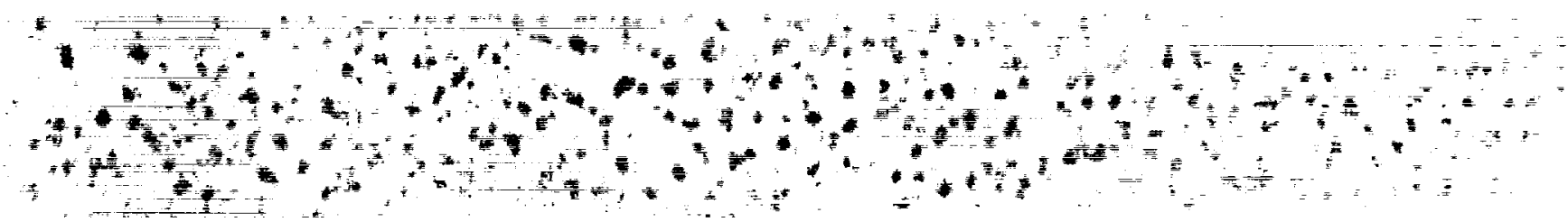




\title{
A FULLY GALERKIN METHOD FOR THE RECOVERY OF STIFFNESS AND DAMPING PARAMETERS IN EULER-BERNOULLI BEAM MODELS
}

\author{
R.C. Smith ${ }^{1}$ \\ Institute for Computer Applications in Science and Engineering \\ NASA Langley Research Center \\ Hampton, VA 23665 \\ K.L. Bowers \\ Department of Mathematical Sciences \\ Montana State University \\ Bozeman, MT 59717
}

\begin{abstract}
A fully Sinc-Galerkin method for recovering the spatially varying stiffness and damping parameters in Euler-Bernoulli beam models is presented. The forward problems are discretized with a sinc basis in both the spatial and temporal domains thus yielding an approximate solution which converges exponentially and is valid on the infinite time interval. Hence the method avoids the time-stepping which is characteristic of many of the forward schemes which are employed in parameter recovery algorithms. Tikhonov regularization is used to stabilize the resulting inverse problem, and the $L$-curve method for determining an appropriate value of the regularization parameter is briefly discussed. Numerical examples are given which demonstrate the applicability of the method for both individual and simultaneous recovery of the material parameters.
\end{abstract}

\footnotetext{
${ }^{1}$ This research was supported by the National Aeronautics and Space Administration under NASA Contract No. NAS1-18605 while the author was in residence at the Institute for Computer Applications in Science and Engineering (ICASE), NASA Langley Research Center, Hampton, VA 23665.
} 


\section{Introduction}

In the modeling and control of large flexible structures, one is often required to numerically recover one or more material parameters given data measurements at various points. Although these structures are in general very complex, in many cases the essential features can be developed by considering a fixed Euler-Bernoulli beam which is assumed to have KelvinVoigt damping. In this paper, a fully Sinc-Galerkin method is presented for the numerical recovery of the stiffness parameter $E I$ and the damping parameter $c_{D} I$ in the state space model

$$
\begin{aligned}
\mathcal{L}\left(E I, c_{D} I\right) u & =f(x, t), \quad 0<x<1, t>0 \\
u(0, t) & =u(1, t)=0, \quad t>0 \\
\frac{\partial u}{\partial x}(0, t) & =\frac{\partial u}{\partial x}(1, t)=0, \quad t>0 \\
u(x, 0) & =\frac{\partial u}{\partial t}(x, 0)=0, \quad 0 \leq x \leq 1
\end{aligned}
$$

with

$$
\mathcal{L}\left(E I, c_{D} I\right) u \equiv \frac{\partial^{2} u}{\partial t^{2}}+\frac{\partial^{2}}{\partial x^{2}}\left(E I(x) \frac{\partial^{2} u}{\partial x^{2}}+c_{D} I(x) \frac{\partial^{3} u}{\partial x^{2} \partial t}\right),
$$

given measurements of the data at the points $\left\{\left(x_{p}, t_{q}\right)\right\}_{p=1, \cdots, n_{p}}^{q=1, \cdots, n_{q}}$ in the domain $(0,1) \times \mathbb{R}^{+}$. From physical considerations, it is reasonable to let the admissible parameter set $Q$ be defined by

$$
Q=\left\{\left(E I, c_{D} I\right) \in \prod_{k=1}^{2} H^{2}(0,1): E I(x) \geq E I_{0}>0, c_{D} I(x) \geq 0\right\}
$$

(see [5]). With this definition, the existence of a unique solution $u$ to the forward problem can be obtained on any fixed time interval $[0, \tau], \tau>0$, for $f$ sufficiently smooth.

The "idealized" parameter recovery problem can then be formulated as follows: determine $q=\left(E I, c_{D} I\right) \in Q$ such that

$$
\mathcal{C} u(\cdot, \cdot, q)=d
$$

where $u(\cdot, \cdot, q)=\mathcal{L}^{-1}(q) f$ denotes the parameter-dependent state solution and $d$ is the data. The observation operator $\mathcal{C}$ is given by

$$
\mathcal{C} \psi=\left\{\psi\left(x_{p}, t_{q}\right)\right\}_{p=1 \cdots n_{p}}^{q=1 \cdots n_{q}}
$$


and hence $\mathcal{C} u$ represents point evaluations of the solution. Note that (1.2) can be written as the operator equation

$$
\mathcal{K}(q)=d
$$

with the nonlinear operator $\mathcal{K}$ given by

$$
\mathcal{K}(q)=\mathcal{C} \mathcal{L}^{-1}(q) f
$$

For reasons similar to those discussed in [11], the problem (1.4) is ill-posed in the sense that solutions $q$ (provided they even exist) may not depend continuously on the data $d$. Consequently, some sort of regularization (i.e., stabilization) is required to obtain an accurate approximation for $q$.

The regularization technique that is used is Tikhonov regularization [13], and the problem (1.4) is replaced by the minimization problem

$$
\min _{\mathbf{q} \in Q} \mathcal{T}_{\alpha}(q)
$$

where

$$
\mathcal{T}_{\alpha}(q) \equiv \frac{1}{2}\left\{\|\mathcal{K}(q)-d\|^{2}+\alpha \mathcal{J}(q)\right\}
$$

Here $\alpha>0$ is a regularization parameter which controls the tradeoff between goodness of fit to the data and stability. The penalty functional $\mathcal{J}(q)$ provides stability and allows the inclusion of a priori information about the true parameters.

Due to the infinite dimensionality both of $Q$ and of the state space, the problem (1.5) is an infinite dimensional minimization problem. In order to develop a practical numerical scheme, the problem must be replaced by a sequence of finite dimensional problems; that is, one must approximate the operator $\mathcal{K}$ and minimize the functional $\mathcal{T}_{\alpha}$ over a finite dimensional admissible subspace of $Q$.

The evaluation of $\mathcal{K}(q)$ requires the solution of (1.1). Similar partial differential equations must be solved to obtain the components of the derivative $\mathcal{K}^{\prime}(q)$. The construction of an approximate solution to these forward problems commonly begins with a Galerkin discretization of the spatial variable with time-dependent coefficients. This yields a system of ordinary differential equations which is solved via differencing techniques. For problems with nontrivial $c_{D} I$, it is noted in [1] that the equations are moderately stiff and routines for 
stiff systems must be employed, thus adding to the expense of the algorithms. This difficulty is further augmented by the fact that the time-stepping must be repeated at each step in the minimization of (1.5). A final difficulty lies in the need to interpolate at data points which do not coincide with the nodes of the ODE solver.

In contrast, the method of this work implements a Galerkin scheme in time as well as space, thus bypassing many of the difficulties associated with time-stepping methods in the context of inverse problems. Corresponding results for the heat equation can be found in [7], and a detailed discussion of fourth-order results involving the recovery of EI in models with no damping is given in [11].

The fully Sinc-Galerkin method in space and time has many salient features due both to the properties of the basis functions and the manner in which the problem is discretized. Perhaps the most distinctive feature of the method is the exponential convergence rate when solving the corresponding forward problems. Furthermore, the judicious choice of a conformal map provides approximate solutions which are valid on the infinite time interval rather than only on a truncated time domain. Finally, the discrete system requires no numerical integrations to fill either the coefficient matrices or the right-hand side matrix. All three features prove to be advantageous when solving the forward problems and hence the inverse problem.

In Section 2, the Sinc-Galerkin system for the forward problem is considered and implementation details are discussed. The forward results are then incorporated into the finite dimensional minimization problems in Section 3 with the discussion centering around the construction of the Tikhonov functional. Numerical results are presented in Section 4 along with a brief outline of the "L-curve" technique for determining the regularization parameter $\alpha$. Examples are given which demonstrate the recovery of the individual parameters $E I$ and $c_{D} I$ as well as the simultaneous recovery of both parameters. 


\section{The Forward Problem}

Consider the forward problem

$$
\begin{aligned}
\mathcal{L} u(x, t) & =f(x, t), \quad 0<x<1, t>0 \\
u(0, t) & =u(1, t)=0, \quad t>0 \\
\frac{\partial u}{\partial x}(0, t) & =\frac{\partial u}{\partial x}(1, t)=0, \quad t>0 \\
u(x, 0) & =\frac{\partial u}{\partial t}(x, 0)=0, \quad 0 \leq x \leq 1
\end{aligned}
$$

with

$$
\mathcal{L} u(x, t) \equiv \frac{\partial^{2} u}{\partial t^{2}}(x, t)+\frac{\partial^{2}}{\partial x^{2}}\left(E I(x) \frac{\partial^{2} u}{\partial x^{2}}(x, t)+c_{D} I(x) \frac{\partial^{3} u}{\partial x^{2} \partial t}(x, t)\right)
$$

Since a thorough derivation of the Sinc-Galerkin method for problems of this type (with $\left.c_{D} I \equiv 0\right)$ is given in [10], the following discussion contains only that material which is needed for the construction of the associated matrix system.

A fully Sinc-Galerkin method for the approximation of the solution of (2.1) may be briefly summarized as follows. For $\phi(x)=\ln \left(\frac{x}{1-x}\right), \Upsilon(w)=\ln (w)$ and positive $h_{x}$ and $h_{t}$, define

$$
\begin{gathered}
S_{i}(x)=S\left(i, h_{x}\right) \circ \phi(x) \equiv \operatorname{sinc}\left(\frac{\phi(x)-i h_{x}}{h_{x}}\right), \\
S_{j}^{*}(t)=S\left(j, h_{t}\right) \circ \Upsilon(t) \equiv \operatorname{sinc}\left(\frac{\Upsilon(t)-j h_{t}}{h_{t}}\right)
\end{gathered}
$$

where

$$
\sin c(x) \equiv \frac{\sin (\pi x)}{\pi x}, \quad-\infty<x<\infty .
$$

The basis is then taken to be $\left\{S_{i j}\right\}$ with

$$
S_{i j}(x, t) \equiv S_{i}(x) S_{j}^{*}(t)
$$

and the approximate solution is defined by way of the tensor product expansion

$$
u_{m_{\mathrm{z}} m_{\mathrm{t}}}(x, t)=\sum_{i=-M_{\mathrm{s}}}^{N_{i}} \sum_{j=-M_{t}}^{N_{t}} u_{i j} S_{i j}(x, t), \quad \begin{aligned}
& m_{x}=M_{x}+N_{x}+1 \\
& m_{t}=M_{t}+N_{t}+1
\end{aligned}
$$


The $m_{x} \cdot m_{t}$ unknown coefficients $\left\{u_{i j}\right\}$ are determined by orthogonalizing the residual with respect to the set of sinc functions $\left\{S_{p} S_{q}^{*}\right\}_{p=-M_{*}, \cdots, N_{*}}^{q=-M_{k}, \cdots, N_{t}}$. This yields the discrete Galerkin system

$$
\left(\mathcal{L} u_{m_{\mathbf{m}} m_{t}}-f, S_{p} S_{q}^{*}\right)=0
$$

for $p=-M_{x}, \cdots, N_{x}$ and $q=-M_{t}, \cdots, N_{t}$. The inner product $(\cdot, \cdot)$ is taken to be

$$
(F, G)=\int_{0}^{\infty} \int_{0}^{1} F(x, t) G(x, t) w(x, t) d x d t
$$

where the weight is

$$
w(x, t)=w(x) w^{*}(t)=\left(\phi^{\prime}(x)\right)^{-\frac{3}{2}}(\dot{\Upsilon}(t))^{-\frac{1}{2}}
$$

A thorough discussion motivating this choice of weight can be found in [10].

The expressions (2.1), (2.7), and (2.8) are then combined to form the system

$$
\begin{aligned}
& \int_{0}^{\infty} \int_{0}^{1} \frac{\partial^{2}}{\partial t^{2}}\left(u_{m_{\bullet} m_{t}}\right) S_{p} S_{q}^{*} w w^{*} d x d t \\
& +\int_{0}^{\infty} \int_{0}^{1} \frac{\partial^{2}}{\partial x^{2}}\left(E I \frac{\partial^{2}}{\partial x^{2}}\left(u_{m_{*} m_{t}}\right)\right) S_{p} S_{q}^{*} w w^{*} d x d t \\
& +\int_{0}^{\infty} \int_{0}^{1} \frac{\partial^{2}}{\partial x^{2}}\left(c_{D} I \frac{\partial^{3}}{\partial x^{2} \partial t}\left(u_{m_{*} m_{t}}\right)\right) S_{p} S_{q}^{*} w w^{*} d x d t \\
& =\int_{0}^{\infty} \int_{0}^{1} f S_{p} S_{q}^{*} w w^{*} d x d t
\end{aligned}
$$

for $p=-M_{x}, \cdots, N_{x}$ and $q=-M_{t}, \cdots, N_{t}$.

In anticipation of the parameter identification problems which motivate this analysis, the terms $E I$ and $c_{D} I$ in (2.9) are expanded as linear combinations of weighted sinc functions with four Hermite-like algebraic terms added to accommodate the potentially nonzero function and derivative values of $E I$ and $c_{D} I$ at $x=0$ and $x=1$. Specifically, this parameter basis is taken to be $\left\{\psi_{k}\right\}_{k=-M_{*}}^{N_{*}}$ with

$$
\psi_{k}(x)= \begin{cases}x(1-x)^{2}, & k=-M_{x} \\ (1-x)^{2}(2 x+1), & k=-M_{x}+1 \\ v_{E}(x) S_{k}(x), & k=-M_{x}+2, \cdots, N_{x}-2 \\ x^{2}(2(1-x)+1), & k=N_{x}-1 \\ -(1-x) x^{2}, & k=N_{x} .\end{cases}
$$


Here $S_{k}(x) \equiv S\left(k, h_{x}\right) \circ \phi(x)$ and the basis weight is taken to be

$$
v_{E}(x)=w(x)=[x(1-x)]^{\frac{3}{2}}
$$

The finite dimensional approximations for $E I$ and $c_{D} I$ are then given by the expansions

$$
E I_{m_{-}}(x)=\sum_{k=-M_{*}}^{N_{k}} c_{k} \psi_{k}(x)
$$

and

$$
c_{D} I_{m_{\bullet}}(x)=\sum_{k=-M_{\bullet}}^{N_{k}} \tilde{c}_{k} \psi_{k}(x) .
$$

In the forward problem, the coefficients $\left\{c_{k}\right\}$ and $\left\{\tilde{c}_{k}\right\}$ are known whereas in the corresponding parameter recovery problems, they are unknown and are determined via methods to be discussed in Section 3. The number of basis functions used in the expansions is chosen so as to guarantee a square spatial coefficient matrix.

A quick note should be made concerning the choice of parameter basis and the manner of expanding $E I_{m_{e}}$ and $c_{D} I_{m_{e}}$. The two derivative-interpolating boundary basis functions are added so that these expansions are the same as those used with cantilever or free boundary conditions. The choice of (2.11) for the basis weight is certainly sufficient and proves to be beneficial when incorporating this forward scheme into a numerical method for solving the parameter recovery problem as described in Section 3.

Sinc quadrature is used to evaluate the integrals in (2.9) and hence derive a discrete system. For details of the quadrature rule and conditions governing its error bound, see [12]; for the purposes of this work, however, it suffices to state the sinc quadrature results as follows. Let $\Gamma$ be $(0,1)$ or $(0, \infty)$ when $\chi=\phi$ or $\Upsilon$, respectively. If $F$ is analytic on $\Gamma$ and suitably bounded, and if there exist positive constants $K, \alpha$, and $\beta$ such that

$$
\left|\frac{F(\tau)}{\chi^{\prime}(\tau)}\right| \leq K \begin{cases}e^{-\alpha|\chi(\tau)|}, & \tau \in \psi((-\infty, 0)) \\ e^{-\beta|\chi(\tau)|}, & \tau \in \psi([0, \infty))\end{cases}
$$

where $\psi=\chi^{-1}$, then for $h>0$ sufficiently small

$$
\left|\int_{\Gamma} F(z) d z-h \sum_{j=-M}^{N} \frac{F\left(z_{j}\right)}{\chi^{\prime}\left(z_{j}\right)}\right| \leq K_{1} e^{-2 \pi d / h}+\frac{K}{\alpha} e^{-\alpha M h}+\frac{K}{\beta} e^{-\beta N h} .
$$

The sinc gridpoints are given by $z_{j}=\psi(j h)=\chi^{-1}(j h)$. 
The expansions (2.12) and (2.13) are substituted into (2.9), and integration by parts is used to transfer the derivatives onto the product $S_{p} w S_{q}^{*} w^{*}$. As detailed in [10], the weight choice (2.8) guarantees that all boundary terms vanish. The resulting integrals are then evaluated via (2.15). For $Y=E I$ or $C_{D} I$ and

$$
\begin{aligned}
\mathcal{Y}(x) \equiv & Y(x)-Y(0)\left[(1-x)^{2}(2 x+1)\right]-Y(1)\left[x^{2}(2(1-x)+1)\right] \\
& -Y^{\prime}(0)\left[x(1-x)^{2}\right]-Y^{\prime}(1)\left[-(1-x) x^{2}\right]
\end{aligned}
$$

the requirement

$$
|\mathcal{Y}(x) u(x, t)| \leq K x^{\alpha+\frac{3}{5}}(1-x)^{\beta+\frac{3}{2}} t^{\gamma+\frac{1}{2}} e^{-6 t}
$$

guarantees the decay needed to truncate the infinite quadrature rule as specified by (2.14). With $\alpha, \beta, \gamma$ and $\delta$ specified and $M_{x}$ chosen, the choices

$$
\begin{gathered}
h_{x}=\sqrt{\frac{\pi d}{\alpha M_{x}}}, \\
h_{t}=h_{x}, \\
N_{x}=\llbracket \frac{\alpha}{\beta} M_{x}+1 \rrbracket, \\
M_{t}=\llbracket \frac{\alpha}{\gamma} M_{x}+1 \rrbracket,
\end{gathered}
$$

and

$$
N_{t}=\llbracket \frac{1}{h_{t}} \ln \left(\frac{\gamma}{\delta} M_{t} h_{t}\right)+1 \rrbracket
$$

for the stepsizes and summation limits balance the asymptotic errors to at least order $\mathcal{O}\left(e^{\left(-\pi d \alpha M_{\infty}\right)^{\frac{1}{3}}}\right)$. This rate results from the presence of a sinc function in the integral. In the above expressions, [.] denotes the greatest integer function. Note that the +1 is unnecessary when $\frac{\alpha}{\beta} M_{x}$ or $\frac{\alpha}{\gamma} M_{x}$ are integers.

Given $M_{x}, N_{x}, M_{t}, N_{t}$ and $h \equiv h_{x}=h_{t}$ as defined above, the discrete system for (2.1) is

$$
A_{E I} U C_{t}^{T}+A_{C_{D} I} U A_{\mathrm{t} 1}^{T}+C_{x} U A_{\mathrm{t} 2}^{T}=G
$$

Here

$$
C_{t} \equiv \mathcal{D}\left(\frac{w^{*}}{\dot{\Upsilon}}\right)
$$




$$
C_{x} \equiv \mathcal{D}\left(\frac{w}{\phi^{\prime}}\right)
$$

and

$$
G \equiv \mathcal{D}\left(\frac{w}{\phi^{\prime}}\right) F \mathcal{D}\left(\frac{w^{*}}{\grave{\Upsilon}}\right)
$$

where $\mathcal{D}$ denotes a diagonal matrix whose entries consist of function evaluations at the sinc gridpoints. The $m_{x} \times m_{t}$ matrices $U$ and $F$ are defined componentwise by

$$
[U]_{i j}=u_{i j}
$$

and

$$
[F]_{i j}=f\left(x_{i}, t_{j}\right) .
$$

It should be noted that the ordering of the coefficients $u_{i j}$ in $U$ mimics that used in most standard time-differencing schemes. This is a matter of convenience since the Sinc-Galerkin method is not bound by any specific ordering of the grid.

As shown in [8], the $m_{t} \times m_{t}$ temporal matrices $A_{t 1}$ and $A_{t 2}$ are given by

$$
\begin{gathered}
A_{t 1}=\left[-\frac{1}{h} I^{(1)}+\frac{1}{2} I\right] \mathcal{D}\left((\dot{\Upsilon})^{\frac{1}{2}}\right), \\
A_{t 2}=\left[\frac{1}{h^{2}} I^{(2)}-\frac{1}{4} I\right] \mathcal{D}\left((\dot{\Upsilon})^{\frac{1}{2}}\right)
\end{gathered}
$$

where $I$ denotes the $m_{t} \times m_{t}$ identity, and $I^{(1)}$ and $I^{(2)}$ are defined componentwise by

$$
\begin{gathered}
{\left[I^{(1)}\right]_{q j}= \begin{cases}0, & j=q \\
\frac{(-1)^{j-q}}{(j-q)}, & j \neq q,\end{cases} } \\
{\left[I^{(2)}\right]_{q j}= \begin{cases}-\frac{\pi^{2}}{3}, & j=q \\
\frac{(-2)(-1)^{j-q}}{(j-q)^{2}}, & j \neq q .\end{cases} }
\end{gathered}
$$

The $m_{x} \times m_{x}$ spatial matrices $A_{E I}$ and $A_{c_{D} I}$ have the form

$$
\begin{aligned}
& A_{E I}=\left[\Phi^{(2)} \mathcal{D}\left(\vec{p}_{\Psi(2)}\right)+2 \Phi^{(3)} \mathcal{D}\left(\vec{p}_{\Psi(1)}\right)+\Phi^{(4)} \mathcal{D}\left(\vec{p}_{\Psi^{(0)}}\right)\right] \\
& A_{C_{\mathcal{D}} I}=\left[\Phi^{(2)} \mathcal{D}\left(\tilde{p}_{\Psi^{(2)}}\right)+2 \Phi^{(3)} \mathcal{D}\left(\tilde{p}_{\Psi^{(1)}}\right)+\Phi^{(4)} \mathcal{D}\left(\tilde{p}_{\Psi^{(0)}}\right)\right]
\end{aligned}
$$


where

$$
\begin{gathered}
\vec{p}_{\Psi(\ell)}=\Psi^{(\ell)} c, \quad \ell=0,1,2, \\
\tilde{p}_{\Psi(\ell)}=\Psi^{(\ell)} \tilde{c}_{,} \quad \ell=0,1,2
\end{gathered}
$$

with $c=\left[c_{-M_{*}}, \cdots, c_{N_{*}}\right]^{T}$ and $\tilde{c}=\left[\tilde{c}_{-M_{\varepsilon}}, \cdots, \tilde{c}_{N_{*}}\right]^{T}$. Finally, the matrices $\Phi^{(j)}, j=2,3,4$ and $\Psi^{(\ell)}, \ell=0,1,2$ are defined componentwise by

$$
\left[\Phi^{(j)}\right]_{p i}=\frac{1}{\phi^{\prime}\left(x_{i}\right)}\left(S_{p} w\right)^{(j)}\left(x_{i}\right)
$$

and

$$
\left[\Psi^{(\ell)}\right]_{i k}=\psi_{k}^{(\ell)}\left(x_{i}\right)
$$

with the notation on the right-hand sides of (2.18) and (2.19) indicating the $j$-th and $\ell$-th derivatives, respectively. The expansions of $\Phi^{(j)}$ and $\Psi^{(\ell)}$ in terms of more fundamental matrices can be found in [10].

For given $E I$ and $c_{D} I$, one then needs to solve the linear system (2.17) for the matrix $U$. As shown on page 414 of [6], (2.17) is algebraically equivalent to

$$
\mathcal{A} \vec{u} \equiv\left\{C_{t} \otimes A_{E I}+A_{t 1} \otimes A_{c_{D} I}+A_{t 2} \otimes C_{x}\right\} \operatorname{co}(U)=c \overrightarrow{o(G)}
$$

where the vector $\vec{u}=c \overrightarrow{o(U)}$ is the concatenation of the $m_{x} \times m_{t}$ matrix $U$ obtained by successively "stacking" the columns of $U$, one upon another, to obtain an $\left(m_{x} \cdot m_{t}\right) \times 1$ vector. The system $(2.20)$ can be solved directly via any of the decomposition methods that are available for large linear systems. It should be noted however, that for large values of $m_{x}$ and $m_{t}$, the ill-conditioning of the $\left(m_{x} \cdot m_{t}\right) \times\left(m_{x} \cdot m_{t}\right)$ matrix $\mathcal{A}$ must be considered when devising schemes for solving the system (2.20). One facet of current research is directed at devising linear algebra techniques which will facilitate the solution of this system. 


\section{The Finite Dimensional Minimization Problem}

As noted in the introduction, the minimization problem

$$
\min _{q \in Q} \mathcal{T}_{\alpha}(q)
$$

where

$$
\mathcal{T}_{\alpha}(q)=\frac{1}{2}\left\{\|\mathcal{K}(q)-d\|^{2}+\alpha \mathcal{J}(q)\right\}
$$

is infinite dimensional and thus must be replaced by a sequence of finite dimensional problems before a viable numerical scheme can be developed. Following from (2.12) and (2.13), the approximating admissible parameter sets are taken to be

$$
\begin{aligned}
Q_{m_{\varepsilon}}=\left\{\left(E I_{m_{\varepsilon}}, c_{D} I_{m_{\varepsilon}}\right):\right. & E I_{m_{\varepsilon}}(x)=\sum_{k=-M_{\varepsilon}}^{N_{\star}} c_{k} \psi_{k}(x), \\
c_{D} I_{m_{\varepsilon}}(x) & \left.=\sum_{k=-M_{\varepsilon}}^{N_{*}} \tilde{c}_{k} \psi_{k}(x)\right\}
\end{aligned}
$$

with the basis $\left\{\psi_{k}\right\}$ defined in (2.10). For $q_{m_{\varepsilon}}=\left(E I_{m_{\varepsilon}}, c_{D} I_{m_{*}}\right)$, the associated finite dimensional optimization problem can then be formulated as

$$
\min _{q_{m_{m}} \in Q_{m_{m}}} \hat{T}_{\alpha}\left(q_{m_{\varepsilon}}\right)
$$

where

$$
\hat{T}_{\alpha}\left(q_{m_{\bullet}}\right) \equiv \frac{1}{2}\left\{\left\|\hat{K}\left(q_{m_{\bullet}}\right)-d\right\|^{2}+\alpha J\left(q_{m_{*}}\right)\right\} .
$$

Note that in solving the minimization problem (3.1), one is actually solving for the vectors $c=\left[c_{-M_{\infty}}, \cdots, c_{N_{\varepsilon}}\right] \in \mathbb{R}^{m_{*}}, \tilde{c}=\left[\tilde{c}_{-M_{*}}, \cdots, \bar{c}_{N_{\bullet}}\right] \in \mathbb{R}^{m_{*}}$, or $\hat{c}=[c, \tilde{c}] \in \mathbb{R}^{2 m_{*}}$ depending on whether one is solving for $E I_{m_{\varepsilon}}, c_{D} I_{m_{\theta}}$, or both parameters simultaneously.

With $n_{p}$ and $n_{q}$ specifying the number of spatial and temporal observation points, respectively, the approximation $\hat{K}\left(q_{m_{\mathbf{E}}}\right)$ to $\mathcal{K}(q)$ is obtained by applying the observation operator $\mathcal{C}$ in (1.3) to $u_{m_{x} m_{t}}$ in (2.5). If the set of observation points $\left\{\left(x_{p}, t_{q}\right)\right\}_{p=1, \cdots, n_{p}}^{q=1, \cdots, n_{q}}$ can be represented as a tensor product of spatial and temporal points, then $\hat{K}\left(q_{m_{*}}\right)$ has the representation

$$
\hat{K}\left(q_{m_{\mathbf{e}}}\right)=C \operatorname{co}(\vec{U})
$$

where the matrix $U$ solves (2.17). $C$ is an $\left(n_{p} \cdot n_{q}\right) \times\left(m_{x} \cdot m_{t}\right)$ evaluation matrix which can be formulated as follows. Define the $n_{p} \times m_{x}$ spatial evaluation matrix $E_{x}$ to have components

$$
\left[E_{x}\right]_{p, i}=S_{i}\left(x_{p}\right), \quad 1 \leq p \leq n_{p}, \quad-M_{x} \leq i \leq N_{x}
$$


and let the $n_{q} \times m_{t}$ temporal evaluation matrix $E_{t}$ have the components

$$
\left[E_{\ell}\right]_{q, j}=S_{j}^{*}\left(t_{q}\right), \quad 1 \leq q \leq n_{q}, \quad-M_{t} \leq j \leq N_{t}
$$

Then

$$
C=E_{t} \otimes E_{x}
$$

It is noted that if the set of observation points is not rectangular as described above, then point evaluation can be done directly via (2.5). This latter option is less efficient, however, than that defined in (3.3).

The form of the discrete penalty functional $J$ depends upon whether one is solving for $E I_{m_{m}}, c_{D} I_{m_{e}}$, or both simultaneously. In the first case, the discrete penalty functional is taken to be

$$
J\left(q_{m_{\bullet}}\right)=\int_{0}^{1}\left[E I_{m_{\bullet}}^{\prime \prime}(x)\right]^{2} d x+\varepsilon \int_{0}^{1}\left[E I_{m_{-}}(x)\right]^{2} d x \approx c^{T} Q c
$$

where the $m_{x} \times m_{x}$ matrix $Q=Q_{d}+Q_{f}$ has components

$$
\left[Q_{d}\right]_{k \ell} \approx \int_{0}^{1} \psi_{k}^{\prime \prime}(x) \psi_{\ell}^{\prime \prime}(x) d x, \quad-M_{x} \leq k, \ell \leq N_{x}
$$

and

$$
\left[Q_{f}\right]_{k \ell} \approx \int_{0}^{1} \psi_{k}(x) \psi_{\ell}(x) d x, \quad-M_{x} \leq k, \ell \leq N_{x}
$$

The exact representations for the matrices $Q_{d}$ and $Q_{f}$ are given in [11]. Similarly, if $E I_{m_{z}}$ is known and $c_{D} I_{m_{\varepsilon}}$ is unknown, an appropriate penalty functional is

$$
J\left(q_{m_{*}}\right)=\int_{0}^{1}\left[c_{D} I_{m_{\varepsilon}}^{\prime \prime}(x)\right]^{2} d x+\varepsilon \int_{0}^{1}\left[c_{D} I_{m_{\varepsilon}}(x)\right]^{2} d x \approx \tilde{c}^{T} Q \tilde{c}
$$

with $\mathrm{Q}$ defined as above. Finally, in the case where both parameters are unknown, the discrete penalty functional can be taken to be

$$
J\left(q_{m_{*}}\right) \approx \hat{c}^{T} Q \hat{c}
$$

where the $2 m_{x} \times 2 m_{x}$ matrix $Q$ is given by

$$
Q=\left[\begin{array}{cc}
Q_{f}+Q_{d} & 0 \\
0 & Q_{f}+Q_{d}
\end{array}\right]
$$


Although the matrix $Q$ in each case is not sparse, as shown in [11] it is very efficient to construct since the component matrices are also needed in the forward solver. Moreover, for $\varepsilon>0$ the matrix $Q$ is symmetric and positive definite and hence has a Cholesky decomposition $Q=R^{T} R$ where $R$ is upper triangular. If $\bar{c}$ is used to denote $c, \bar{c}$, or $\hat{c}$, then it follows that the penalty term $J\left(q_{m_{e}}\right)$ yields the quadratic form

$$
\bar{c}^{T} R^{T} R \bar{c}=\|R \bar{c}\|^{2}
$$

where $\|\cdot\|$ denotes the Euclidean norm. This representation for the penalty functional is particularly useful both when implementing a scheme to solve the minimization problem and when plotting the "L-curve" to determine a suitable regularization parameter $\alpha$ (see [3]).

To highlight the dependence of the functional $\hat{T}_{\alpha}$ in (3.2) on the unknown vector $\bar{c}$, let

$$
K(\bar{c}) \equiv \hat{K}\left(q_{m_{\bullet}}\right)=C \operatorname{co}(\vec{U}(\bar{c}))
$$

where $U(\bar{c})$ solves (2.17) for a given $\bar{c}$. Noting (3.4), the optimization problem (3.1) can be replaced by

$$
\min _{\bar{c}} T_{\alpha}(\bar{c})
$$

where

$$
T_{\alpha}(\bar{c})=\frac{1}{2}\left\{\|K(\bar{c})-d\|^{2}+\alpha\|R \bar{c}\|^{2}\right\} .
$$

To obtain a minimizer for the nonlinear functional $T_{\alpha}(\bar{c})$, a quasi-Newton trust region iteration [2]

$$
\bar{c}_{k+1}=\bar{c}_{k}+s_{k}
$$

is used. Here $s_{k}$ solves the quadratic programming problem

$$
\min \frac{1}{2}\left\{\left\|K\left(\bar{c}_{k}\right)+K^{\prime}\left(\bar{c}_{k}\right) s-d\right\|^{2}+\alpha\left\|R\left(\bar{c}_{k}+s\right)\right\|^{2}\right\}
$$

subject to $\|s\| \leq \delta_{k}$ with $K^{\prime}\left(\bar{c}_{k}\right)$ denoting the Jacobian of $K$ at $\bar{c}_{k}$. The trust region radius is chosen so that $T_{a}(\bar{c})$ has sufficient decrease at each iteration to guarantee convergence to a local minimizer of $T_{\alpha}$ (for further details about the theory and implementation of the trust region algorithm, see [2] or [4]). 
An important numerical issue in the implementation of the trust region scheme is the formulation of the derivative of the operator $K$. Here the derivative, or Jacobian, is the matrix whose $\nu$-th column is given by

$$
\left[K^{\prime}(\bar{c})\right]_{\nu}=\lim _{\mathcal{T} \rightarrow 0} \frac{1}{\mathcal{T}}\left[K\left(\bar{c}+\mathcal{T} e_{\nu}\right)-K(\bar{c})\right]
$$

where the standard unit vector $e_{\nu}$ has components

$$
\left[e_{\nu}\right]_{k}=\delta_{\nu k}= \begin{cases}1, & k=\nu \\ 0, & k \neq \nu\end{cases}
$$

In the examples of the next section, the Jacobians are calculated with a standard forward difference scheme. This scheme is easy to implement and accurate enough for the purposes of the method. If further efficiency is desired, a directional derivative scheme such as that described in [7] can be used.

\section{Implementation and Numerical Examples}

The three examples in this section demonstrate the use of the Sinc-Galerkin method for recovering the individual parameters $E I$ and $c_{D} I$ as well as the simultaneous recovery of both parameters. In each case the state solution is $u(x, t)=x(1-x) \sin (4 \pi x) t^{2} e^{-t}$ and the true material parameters are $E I(x)=1+x+x(1-x)$ and $c_{D} I(x)=1+\sin (\pi x)$. For these functions, the choices $\alpha=\beta=\gamma=\frac{3}{2}$ and $\delta=1$ satisfy the decay condition (2.16). In all three cases, the dynamics of the problem are assumed to be modeled by (1.1) with the forcing function $f(x, t)$ consistent with the state solution and the true stiffness and damping parameters. 
In the first example, $E I$ is considered known and $c_{D} I$ is numerically recovered using the methods of this paper. The roles are reversed in the second example and $E I$ is numerically recovered while $c_{D} I$ is considered known. The final example demonstrates the numerical recovery of both unknown parameters. It should be noted that a comprehensive set of examples demonstrating the application of the Sinc-Galerkin method to models of undamped beams $\left(c_{D} I \equiv 0\right)$ can be found in [11].

A very important practical consideration is the choice of the regularization parameter $\alpha$ for a given (error-contaminated) data set. If the error in the data is discrete and random, then under certain conditions the method of Generalized Cross Validation (GCV) can be used to determine a suitable value of $\alpha$ (see [14]). A second method for determining the regularization parameter is to plot the norm of the penalty functional, $\left\|R \bar{c}_{\alpha}\right\|$, versus the norm of the residual, $\left\|K\left(\bar{c}_{\alpha}\right)-d\right\|$ (see [3] or [9]). Here $\bar{c}_{\alpha}$ denotes the solution to (3.5). In this way, one can qualitatively get an idea of the compromise between the minimization of these two quantities. The scheme for determining the "optimal" regularization parameter consists of finding those values of $\alpha$ such that $\left(\left\|K\left(\bar{c}_{\alpha}\right)-d\right\|,\left\|R \bar{c}_{\alpha}\right\|\right)$ lies in the "corner" of the resulting curve, known as the $L$-curve. The use of this technique for determining suitable choices for the regularization parameter is demonstrated in the examples.

In each of the three examples, the data was sampled on a regular grid $\left\{\left(x_{p}, t_{q}\right)\right\} \subset$ $(0,1) \times(0,3]$. Nine equally spaced points $x_{p}=p \Delta x, \Delta x=.1$, were taken in space and six equally spaced temporal points $t_{q}=q \Delta t, \Delta t=.5$, were taken for a total of $n=54$ data points. To the data, we added a pseudo-random noise vector $\varepsilon$ from a Gaussian distribution with mean 0 and standard deviation $\sigma$ chosen so that the noise-to-signal ratio $\sigma /\|d\|=.001$; that is, noise $=.1 \%$ of the signal.

The summation limits were taken to be $M_{x}=N_{x}=M_{t}=8$ and $N_{t}=4$ as dictated by the choice of decay parameters. Hence $m_{x}=17$ basis functions were used in the expansion of each material parameter. The startup vectors in each example were chosen to reflect the positivity and general endpoint behavior of the true parameters. All problems were run with sixteen place accuracy on a Vax 8550. 


\section{Example 4.1.}

In this example, the parameter $E I$ is considered to be known and the parameter $c_{D} I$ is to be numerically recovered. The startup vector was taken to be the $m_{x} \times 1$ vector $\bar{c}_{0}=[2, .5, .5, \cdots, .5, .5,-2]^{T}$. For varying values of the regularization parameter $\alpha$, the L-curve is plotted in Figure 4.1. Note that the points $\alpha=10^{-5}$ and $\alpha=5 \times 10^{-6}$ yield points $\left(\left\|K\left(\bar{c}_{\alpha}\right)-d\right\|,\left\|R \bar{c}_{\alpha}\right\|\right)$ in the "corner" of the curve. For $\alpha=5 \times 10^{-4}, \alpha=10^{-5}$ and $\alpha=10^{-7}$, the plots of the true and approximate damping parameters are given in Figure 4.2. It can be seen that the "corner" value, $\alpha=10^{-5}$, provides a good choice for the regularization parameter whereas $\alpha=10^{-7}$ is not large enough to damp out the contribution due to the smaller singular values. Finally, the choice $\alpha=5 \times 10^{-4}$ causes too much smoothing and information about the parameter is lost. The results from this example demonstrate the viability of the method for problems in which $E I$ is known and $c_{D} I$ is unknown.

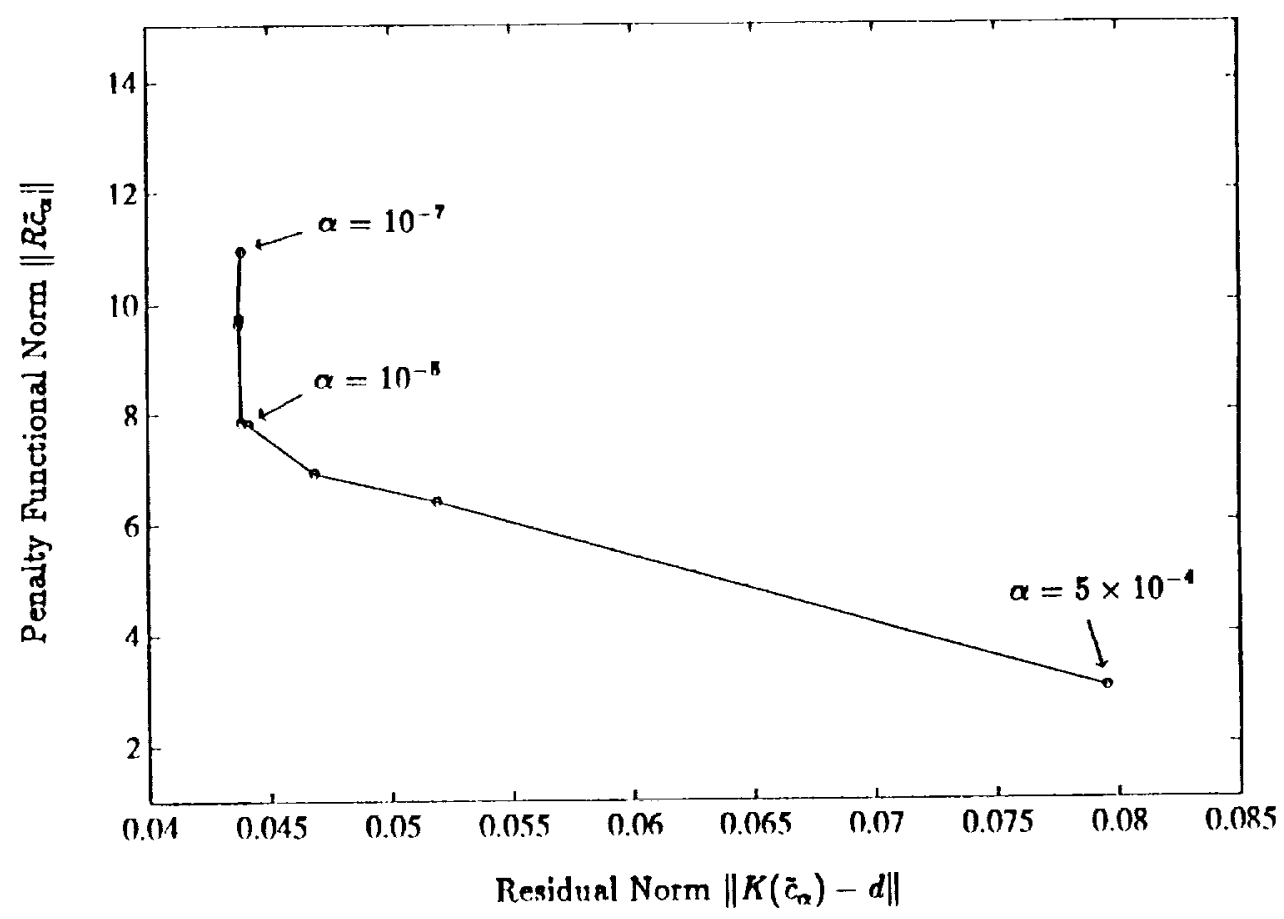

Figure 4.1: The Tikhonov $L$-Curve for Example 4.1. 


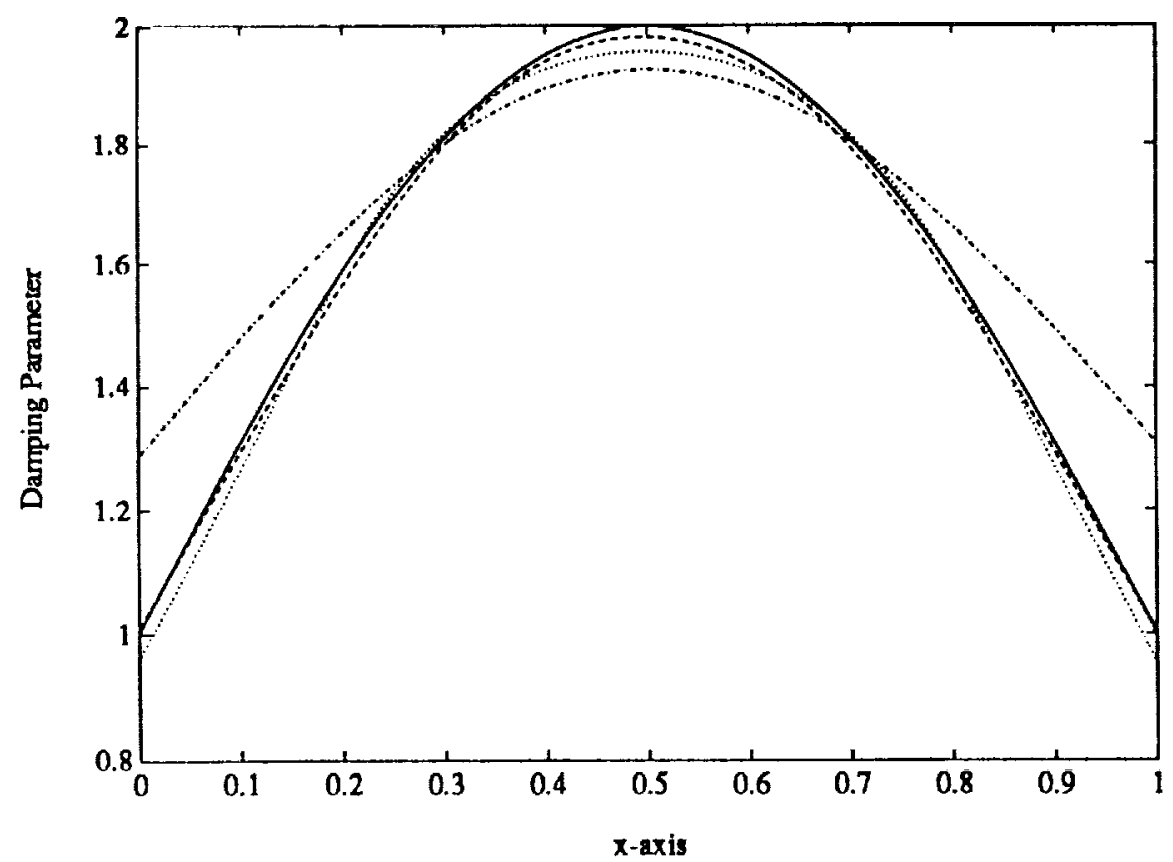

Figure 4.2: True and Approximate Damping Parameters for Example 4.1

$$
-\cdots\left(\alpha=5 \times 10^{-4}\right),--\left(\alpha=10^{-5}\right), \cdots\left(\alpha=10^{-7}\right),- \text { (True). }
$$

\section{Example 4.2}

Consider now the case where the stiffness parameter $E I$ is considered to be unknown and the damping parameter $c_{D} I$ is assumed to be known. Here the $m_{x} \times 1$ startup vector $c_{0}=[1,1, .5, \cdots, .5,2,0]^{T}$ was used. Since the L-curve for this example was very similar to that in Example 4.1, the true and approximate stiffness parameters corresponding to $\alpha=5 \times 10^{-4}, \alpha=10^{-5}$ and $\alpha=10^{-7}$ were computed with results given in Figure 4.3. It is again noted that the "corner" value of $\alpha=10^{-5}$ provides a good choice for the regularization parameter whereas the choice $\alpha=5 \times 10^{-4}$ causes too much smoothing. Finally, the error contributions due to the smaller singular values become more apparent with $\alpha=10^{-7}$. 


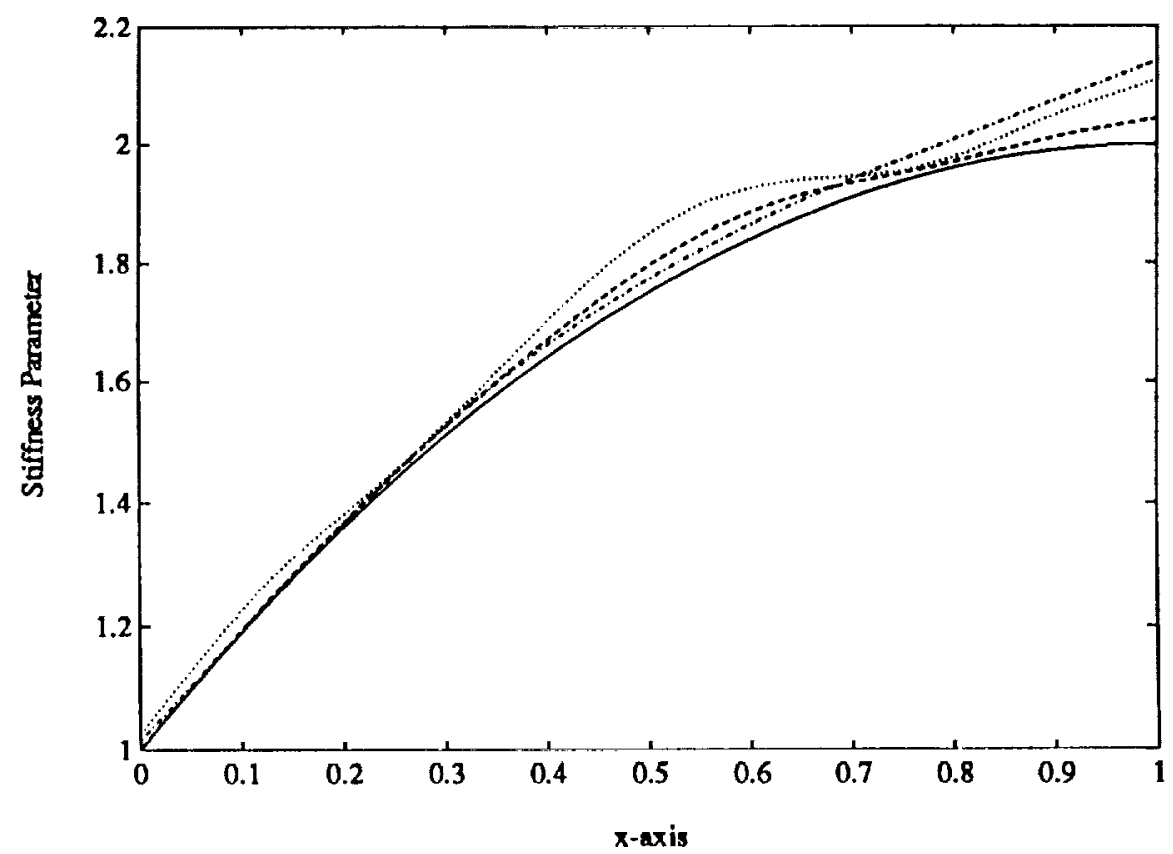

Figure 4.3: True and Approximate Stiffness Parameters for Example 4.2

$$
-\cdot\left(\alpha=5 \times 10^{-4}\right),---\left(\alpha=10^{-5}\right), \cdots\left(\alpha=10^{-7}\right),- \text { (True). }
$$

\section{Example 4.3}

In this example, the method is used to simultaneously recover both the stiffness parameter $E I$ and the damping parameter $c_{D} I$. Following from the previous two examples, the $2 m_{x} \times 1$ initial vector had the form $\hat{c}_{0}=\left[c_{0}, \tilde{c}_{0}\right]^{T}$. The true and approximate material parameters corresponding to $\alpha=10^{-5}$ are plotted in Figures 4.4 and 4.5. From the figures it is noted that although the method is accurately recovering the shape of the functions, there is more error in the magnitude than in the previous two examples. Hence it appears that a larger number of state and parameter basis functions are needed to accurately recover both parameters simultaneously, and current efforts are directed at devising linear algebra techniques which would facilitate the solution of the larger discrete systems. 


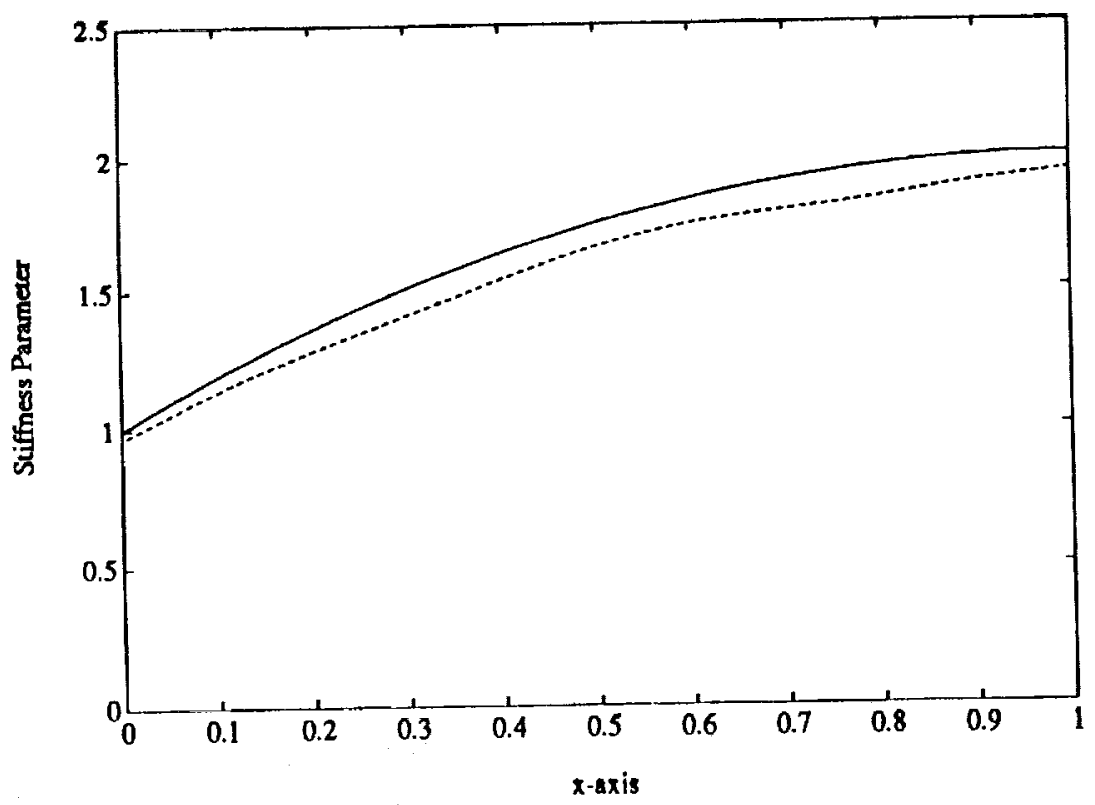

Figure 4.4: True and Approximate Stiffness Parameters for Example 4.3

$$
---\left(\alpha=10^{-5}\right) \text {, - (True). }
$$

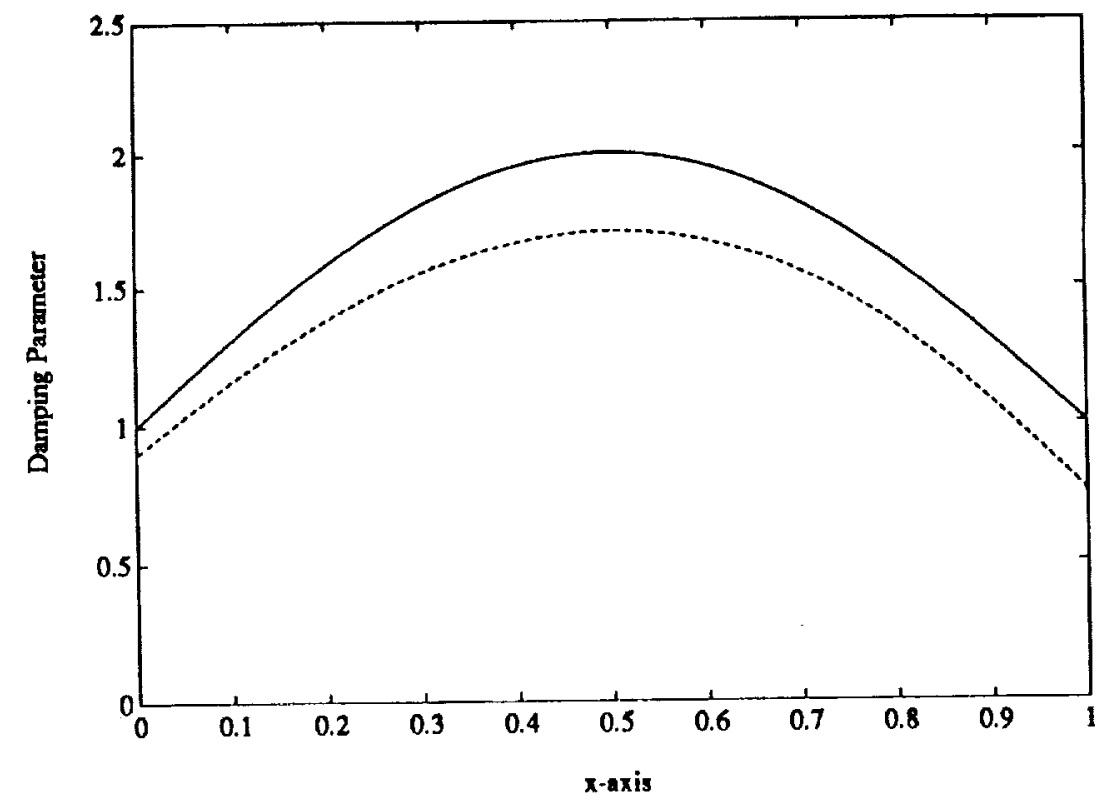

Figure 4.5: True and Approximate Damping Parameters for Example 4.3

$$
---\left(\alpha=10^{-5}\right),-(\text { True }) \text {. }
$$




\section{Acknowledgement}

The authors would like to thank the staff of the Department of Mathematical Sciences at Montana State University for the many times they provided assistance before, during and after this conference.

\section{References}

[1] H.T. Banks, J.M. Crowley and I.G. Rosen, "Methods for the Identification of Material Parameters in Distributed Models for Flexible Structures," Mat. Aplic. Comp., v. 5,1986 , pp. $139-168$.

[2] J.E. Dennis and R.B. Schnabel, Numerical Methods for Unconstrained Optimization and Nonlinear Equation, Prentice Hall, Englewood Cliffs, NJ, 1983.

[3] P.C. Hansen, "Analysis of Discrete Ill-Posed Problems by Means of the L-curve," submitted to SIAM Rev.

[4] K. Jonca and C.R. Vogel, "Numerical Solution to the Magnetic Relief Problem," Transport Theory, Invariant Imbedding, and Integral Equations (Lecture Notes in Pure and Applied Mathematics), ed. P. Nelson et al, Marcel Dekker, New York, 1989, pp. 379-391.

[5] K. Kunisch and E. Graif, "Parameter Estimation for the Euler-Bernoulli Beam," Mat. Aplic. Comp., v. 4, 1985, pp. 95-124.

[6] P. Lancaster and M. Tismenetsky, The Theory of Matrices, Academic Press, Orlando, FL, 1985.

[7] J. Lund and C.R. Vogel, "A Fully-Galerkin Method for the Solution of Inverse Problems in Parabolic Partial Differential Equations," Inv. Prob., v. 6, 1990, pp. 205-217.

[8] K.M. McArthur, K.L. Bowers and J. Lund, "The Sinc Method in Multiple Space Dimensions: Model Problems," Numer. Math., v. 56, 1990, pp. 789-816. 
[9] K. Miller, "Least Squares Methods for Ill-Posed Problems with a Prescribed Bound," SIAM J. Math. Anal., v. 1, 1970, pp. 52-74.

[10] R.C. Smith, K.L. Bowers and J. Lund, "A Fully Sinc-Galerkin Method for EulerBernoulli Beam Models," submitted to Numer. Methods Partial Diff. Equations.

[11] R.C. Smith, K.L. Bowers and C.R. Vogel, "Numerical Recovery of Material Parameters in Euler-Bernoulli Beam Models," submitted to J. Math. Systems and Control.

[12] F. Stenger, "A Sinc-Galerkin Method of Solution of Boundary Value Problems," Math. Comp., v. 33, 1979, pp. 85-109.

[13] A.N. Tikhonov and V.Y. Arsenin, Solutions of Ill-Posed Problems, Wiley, New York, 1977.

[14] G. Wahba, "Practical Approximate Solutions to Linear Operator Equations when the Data are Noisy," SIAM J. Numer. Anal., v. 14, 1977, pp. 651-667. 


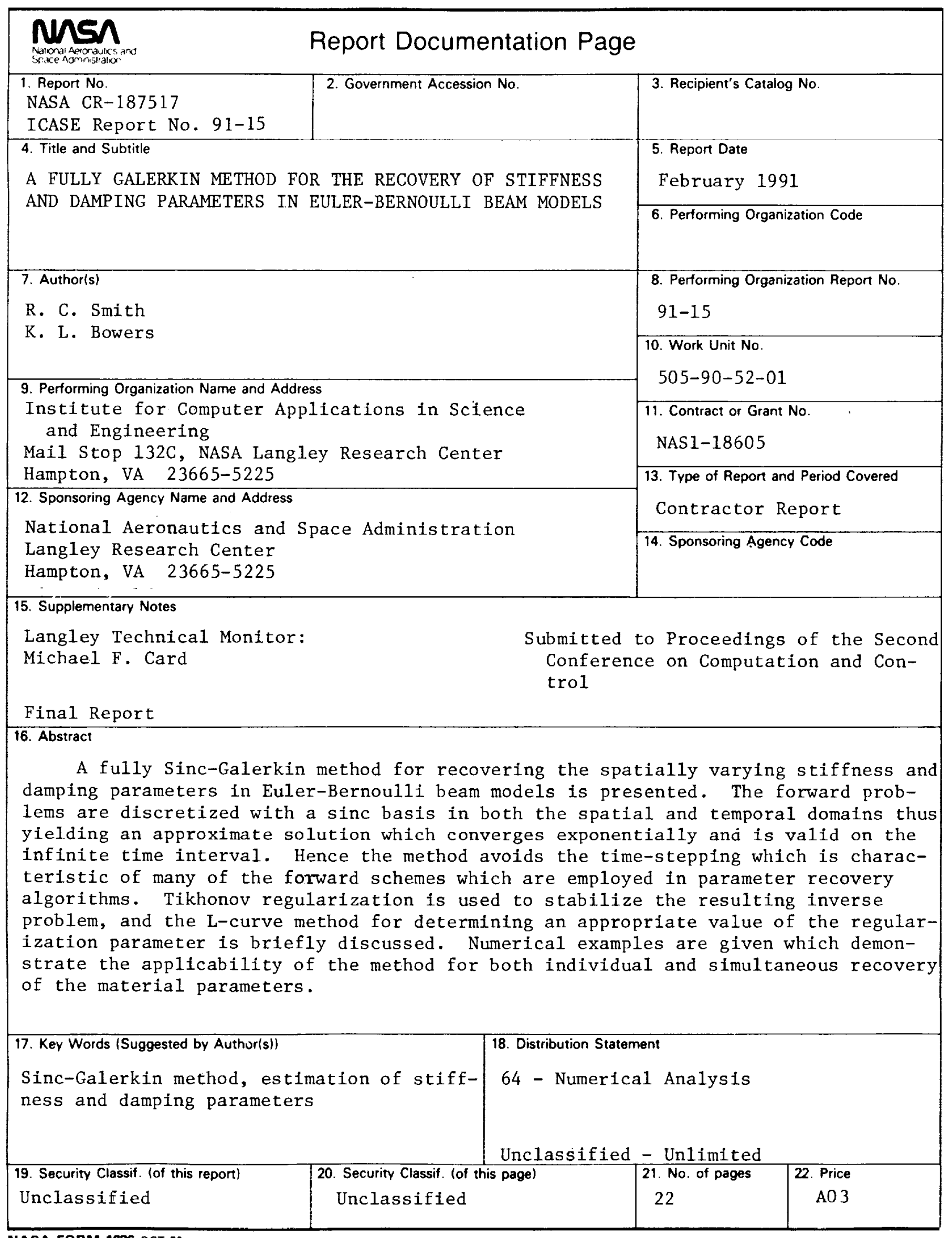


